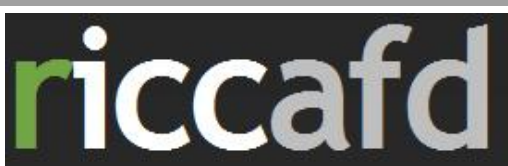

Revista Iberoamericana de Ciencias de la Actividad Física y el Deporte

\title{
RELACIÓN DEL ÍNDICE DE MASA CORPORAL Y EL PORCENTAJE DE GRASA CORPORAL EN JÓVENES VENEZOLANOS
}

\section{RELATIONSHIP OF BODY MASS INDEX AND BODY FAT PERCENTAGE IN YOUNG VENEZOLANOS}

\section{MSc. José Padilla}

Unidad Educativa de Talento Deportivo del Estado Barinas, Barinas, Venezuela

Correo electrónico: joserafael.pa@gmail.com

Código UNESCO: 2402.05

Clasificación Consejo de Europa: 9

Recibido el 30 de marzo de 2014

Aceptado el 12 de abril de 2014

PALABRAS
CLAVE:
Sobrepeso,
Obesidad,
Adiposidad,
Porcentaje de
grasa,
Indice de Masa
Corporal

\section{RESUMEN}

El índice masa corporal (IMC) es uno de los criterios mayormente utilizados para definir la obesidad en la población infantil. No obstante, el mismo determina el estado nutricional, mientras que el total de la grasa corporal demarca su composición corporal. Por lo tanto, el objetivo general del estudio fue determinar la relación entre el índice de masa corporal y el porcentaje de grasa corporal en 505 estudiantes (242 del sexo femenino y 263 masculino) de un Liceo Bolivariano del estado Barinas, con edades comprendidas entre 10 y 20 años. La investigación se enmarcó en el enfoque cuantitativo, con un tipo de investigación de campo, basado en un diseño no experimental, de nivel descriptivo-correlacional y de corte transversal. El análisis de datos se realizó a través del software estadístico SPSS versión 17.0. Los resultados presentan una correlación alta entre el IMC y el porcentaje de grasa $\left(\mathbf{r}: \mathbf{0 , 7 5 ^ { \star \star }}\right.$ para las chicas y r: $\mathbf{0 , 7 7 ^ { \star * }}$ para los chicos; $\left.\mathbf{p}<0,01\right)$. Asimismo, se presentaron correlaciones altas y significativas en los diferentes grupos de edad para ambos sexos. Para el masculino G1: $r$ : 0,81, p<0,01; G2: $r$ : $0,82^{\star \star}, p<0,01$ y G3: $r: 0,66^{\star \star}, p<0,01$. En el caso del femenino la relación fue G1: $r: 0,72^{\star \star} ; G_{2}: r: 0,83^{\star \star}$ y G3: $r$ : $\mathbf{0 , 8 1 ^ { \star * }}, \mathbf{p}<\mathbf{0 , 0 1}$. Se concluye de esta manera que existe alta correlación positiva entre el IMC y el porcentaje de grasa corporal en la Institución Educativa mencionada, por lo tanto se considera la utilización del IMC como una herramienta aplicable para la estimación del estado nutricional.

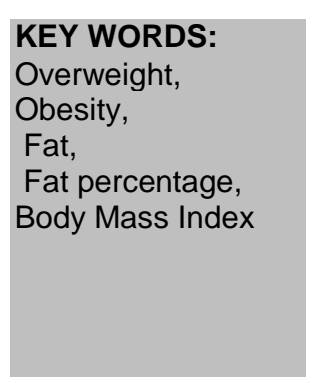

\section{ABSTRACT}

The body mass index (BMI) is one of the mostly used criteria to define obesity in children. However, it determines the nutritional status, while the total body fat demarcates body composition. Therefore, the objective of the study was to determine the relationship between body mass index and body fat percentage in 505 students (242 female and 263 male) of Barinas state Bolivarian High School, aged between 10 and 20 years. The research was part of the quantitative approach, a kind of field research, based on a non-experimental, correlational descriptive level and cross section. Data analysis was performed using SPSS statistical software version 17.0. The results show a high correlation between BMI and body fat percentage ( $r: 0,75^{* *}$ for girls and $r$ : $0,77^{* *}$ for boys, $\left.p<0,01\right)$. Also showed high and significant correlations in different age groups for both sexes. For the male G1: $\mathbf{r}: \mathbf{0 , 8 1 ,} \mathbf{p}$ $<0.01, \mathrm{G2}: \mathrm{r}: 0,82{ }^{\star *}, \mathrm{p}<0.01$ and G3: $r: 0,66^{* *}, \mathrm{p}<0.01$. In the case of women the relationship was G1: $r: 0,72{ }^{\star \star}$; G2: $\mathbf{r}: \mathbf{0 , 8 3}{ }^{\star *}$ and G3: $\mathbf{r}: \mathbf{0 , 8 1}{ }^{\star *}, \mathbf{p}<\mathbf{0 . 0 1}$. It thus concludes that there is high positive correlation between BMI and body fat percentage in the educational institution mentioned therefore considered the use of BMI as a tool applicable for estimating nutritional status. 


\section{INTRODUCCIÓN}

El sobrepeso y la obesidad representan en la actualidad problemas de salud pública, tanto en países desarrollados, como en los que se encuentran en vías del desarrollo. Sobre este particular ${ }^{(1)}$, mencionan que "su presencia en edades tempranas es factor de riesgo de obesidad y de riesgo coronario en la edad adulta; de ahí la importancia de prevenirla, detectarla y tratarla oportunamente" (p. 1). Por su parte ${ }^{(2)}$, ha destacado que la obesidad ha alcanzado proporciones epidémicas a nivel mundial, y cada año mueren, como mínimo 2,6 millones de personas a causa de la obesidad o sobrepeso.

En el ámbito Venezolano, y de acuerdo al más reciente ranking, hecho por ${ }^{(3)}$ la prevalencia de obesidad se ubica alrededor del $31 \%$, lo cual indica que 3 de cada 10 de ellos son obesos. Por su parte, en el caso de las edades infantiles (4), en un estudio realizado en 18.000 niñas, niñas y adolescentes con edades entre 7 y 17 años, Venezuela registró $9 \%$ de incidencia de obesidad en la población anteriormente descrita, estando este porcentaje muy cercano al $10 \%$ que establece la OMS como límite mínimo para decretar un problema de salud pública.

Lo anteriormente destaca la preocupación de estos organismos en lo referente a considerar a la obesidad como un problema de salud publica. Dentro de este marco, es necesario destacar que para lograr un diagnóstico adecuado de sobrepeso y obesidad se hace indispensable el poder contar con indicadores antropométricos que se relacionen con la obesidad.

Sobre este particular, la Organización Mundial de la Salud ${ }^{(2)}$, ha definido a la obesidad "como la condición en la cual el exceso de tejido adiposo afecta de manera adversa la salud y el bienestar" (p. 5). En ese mismo sentido (5), destacan lo siguiente: "para diagnosticar correctamente la obesidad, debemos además de constatar un peso elevado, evaluar si este es debido a un aumento de la masa grasa; para ello tenemos que analizar la composición corporal" (p. 283). Según lo anteriormente descrito, los indicadores que mejor predicen la obesidad son aquellos que cuantifiquen el tejido adiposo, y por consiguiente, la estimación del porcentaje de grasa corporal.

Existen diversos métodos que permiten evaluar la composición corporal, presentando cada uno de estos ventajas y desventajas en lo referente al grado de precisión, costo y practicidad para el personal que los realiza. Dentro de estos se tiene a la absorciometría por rayos $\mathrm{x}$ de doble energía, la densitometría (contando con 2 métodos: el pesado hidrostático y la plestimografia por desplazamiento de aire), la bioempedancia eléctrica y la antropometría. Sin embargo, algunos de ellos resultan imprácticos tanto por sus altos costos como por su rigurosa formación del personal que los maneje. Por lo tanto, la obesidad en el adulto ha sido cuantificada por indicadores que midan solo la masa corporal, tal es el caso de la antropometría.

El índice masa corporal (IMC) o índice de Quételet, el cual resulta de dividir el peso corporal en kilogramos entre el cuadrado de la estatura en metros, es uno de los criterios mayormente utilizados por los investigadores para definir el sobrepeso y la obesidad en la población adulta, y en la actualidad se recomienda en población pediátrica. Asimismo, es comúnmente empleado como uno de los indicadores para valorar el estado nutricional, el cual se correlaciona con la masa grasa corporal y el riesgo de complicaciones cardiovasculares asociadas a la obesidad. Sin embargo, tal como lo señalan ${ }^{(6)}$, el IMC también refleja la masa libre de grasa, la masa muscular y la masa ósea, lo cual resulta en numerosas variaciones de estos componentes del cuerpo dentro de la misma masa corporal. No obstante, la facilidad de la toma de estas variables antropométricas básicas, con bajo error y buena reproducibilidad ofrece la posibilidad a través de índices simples (IMG o, en su defecto, IMC) de la valoración de la adiposidad corporal total desde un punto de vista clínico, lo que constituye una herramienta de valiosa importancia para la clasificación diagnostica del sobrepeso de los adolescentes españoles ${ }^{(7)}$.

De igual forma ${ }^{(8)}$, mencionan que aunque el índice de masa corporal $\left(\mathrm{kg} / \mathrm{m}^{2}\right)$ se utiliza ampliamente como un índice de la grasa corporal, es una medida de peso en relación con la altura, en lugar que de la adiposidad. Por su parte ${ }^{(9)}$, en su estudio determinaron que los variaciones en el IMC durante el crecimiento reflejan cambios en el tamaño y numero de adipocitos, aumentando desde el nacimiento hasta 1 año, luego disminuye gradualmente y alcanza un mínimo a los 5-6 años, luego comienza aumentar durante el resto de la infancia. Sin embargo ${ }^{(10)}$, sugieren que los cambios en el IMC no pueden reflejar con precisión los cambios en la adiposidad.

En líneas generales, los estudios anteriormente destacados coinciden en la utilización del IMC como indicador para la valoración del estado nutricional. Sin embargo, existen ciertas inconsistencias en su utilidad para el análisis de la composición corporal. Sobre la base de este aspecto y aunado a la falta de investigaciones

Rev. lb. Cᄃ. Act. Fis. Dep. 
Padilla, J.

en el ámbito regional que relacionen el IMC con la composición corporal, han llevado a la realización del presente trabajo.

En tal sentido el objetivo general es determinar la relación entre el IMC y el porcentaje de grasa corporal en estudiantes con edades entre $10 \mathrm{y}$ 20 años.

\section{MATERIAL Y MÉTODOS}

\section{Caracterización de la investigación}

El estudio se enmarcó en el enfoque cuantitativo, con un tipo de investigación de campo, basado en un diseño no experimental, de nivel descriptivo-correlacional y de corte transversal. La muestra utilizada equivale al total de la población, la cual incluyó a 505 estudiantes (263 del sexo masculino y 242 del sexo femenino), con edades comprendidas entre 10 y 20 años. Los sujetos fueron divididos en 3 subgrupos de edad (G1: < 13,52; G2: 13,52 - 15,19 y G3: > 15,19), tomando en consideración la estrategia de recodificación de variables a partir de un análisis de frecuencias.

\section{Protocolos para la recopilación de los datos}

El protocolo utilizado para las mediciones antropométricas, obedece a los estándares establecidos por la Sociedad Internacional para

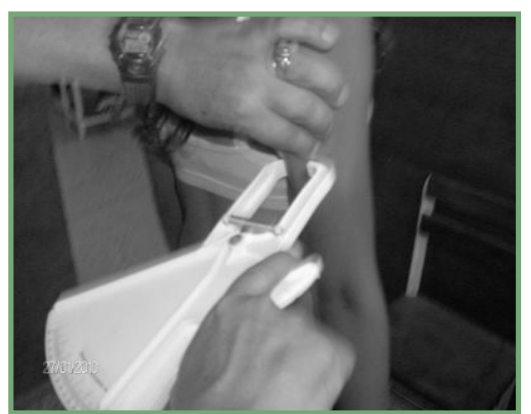

Figura 1. Medición pliegue del tríceps.

\section{Modelo estadístico}

Se utilizó la prueba de Kolmogorov-Smirnov para comprobar que las variables no se alejaban significativamente del comportamiento normal. Sobre este particular ${ }^{(12)}$, señala que "conocer la forma de distribución de la variable es importante para elegir la prueba adecuada; por ello en muchas ocasiones es imprescindible utilizar pruebas previas que permitan decidir qué técnica, prueba, test o contraste se utilizará en el análisis" (p.108).

Rev. Ib. CL. Act. Fis. Dep. el Avance de la Kinantropometría (I.S.A.K. - The International Society for the Advancement of Kinanthropometry). Se les indicó a los estudiantes acudir a la sala de evaluación con pantalón corto o licra corta, con el objetivo de facilitar las mediciones. Todas las medidas fueron tomadas por el autor del presente trabajo, quien cuenta con acreditación nivel II expedido por la ISAK. Se contó con un error técnico de medida dentro de los márgenes aceptados por la organización internacional anteriormente mencionada.

Se tomaron las medidas siguientes: la estatura parada y masa corporal, para con estas variables calcular el índice de Masa Corporal (IMC), dividiendo el peso en kilogramos entre la estatura en metros elevada al cuadrado $\left(\mathrm{kg} / \mathrm{m}^{2}\right)$ y para clasificar a los estudiantes con sobrepeso y obesidad, se usaron los puntos de corte sugeridos por el Grupo de Trabajo sobre Obesidad (IOTF - International Obesity Task Force) para niños y adolescentes por edad y sexo.

Asimismo, se midieron los pliegues del tríceps (ver figura 1) y subescapular (ver figura 2) utilizando un calibrador de pliegues marca Slim Guide con $1 \mathrm{~mm}$ de precisión, para calcular el porcentaje de grasa (\% grasa) utilizando la fórmula ${ }^{(11)}$

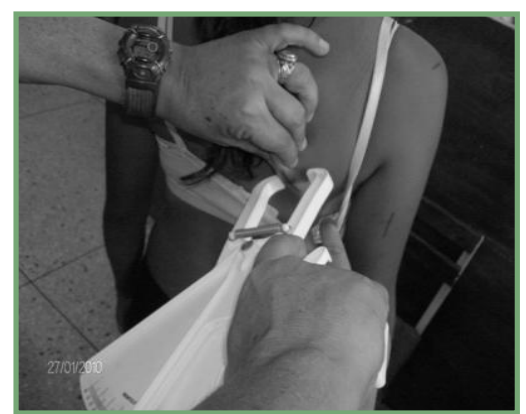

Figura 2. Medición pliegue subescapular

El segundo análisis llevado a cabo es el descriptivo, donde se calcularon medias, valores máximos y mínimos, desviación típica, en todas las variables de estudio. Para verificar la relación entre el IMC y el \% grasa se empleó el coeficiente de correlación de Pearson (r), utilizándose para valorar su magnitud de relación la escala ${ }^{(13)}$, siendo $r$ : muy baja $(0,00$ $0,20)$, baja $(0,21-0,40)$, moderada $(0,41-0,60)$, alta $(0,62-0,80)$ y muy alta $(0,81-1,00)$. El tercer análisis consistió en determinar la varianza explicada $\left(r^{2}\right)$, entre las variables estudiadas, que indicó el porcentaje de relación compartida 
en escala de 0 - 100\%, que existe entre las mismas, para ambos sexos.

Los análisis se realizaron con el paquete estadístico para las ciencias sociales (SPSS Statistical Package for the Social Sciences) versión 17.0 para Windows. Todos los análisis estadísticos se han realizado con un nivel de significación estadística de $p<0,05$; para garantizar una confianza del $95 \%$ en la aseveración de cada conclusión.

\section{RESULTADOS}

En la tabla 1 se pueden ver los estadísticos descriptivos para las variables estudiadas, apreciándose diferencias significativas entre los sexos específicamente en la estatura y el porcentaje de grasa corporal, resultando valores superiores para el sexo masculino en la primera variable y en el femenino para la segunda.

Tabla 1. Características antropométricas de los sujetos evaluados.

\begin{tabular}{lccccc} 
& \multicolumn{2}{c}{$\begin{array}{c}\text { MASCULINO } \\
\text { (N: 263) }\end{array}$} & \multicolumn{3}{c}{$\begin{array}{c}\text { FEMENINO } \\
\text { (N: 242) }\end{array}$} \\
\hline Edad (años) & $\mathbf{X}$ & DS & $\mathbf{X}$ & $\mathbf{D S}$ & t student \\
Masa corporal (kg) & 14,58 & 1,82 & 14,36 & 1,65 & NS \\
Estatura (cms) & 49,240 & 12,860 & 47,665 & 8,210 & NS \\
IMC (kg/m2) & 159,72 & 11,09 & 154,79 & 6,12 & $<0,05$ \\
\% Grasa (\%) & 21,05 & 3,16 & 21,69 & 2,99 & NS \\
\hline
\end{tabular}

NS: No significativo

Por otra parte, en la tabla 2 se destacan las correlaciones entre las variables estudiadas, obteniéndose en la población masculina la correlación más alta en los sujetos con peso ideal, tendencia semejante sucedió en el sexo femenino. Sin embargo, la distinción se vislumbra en la correlación entre el IMC y el \% de grasa, donde ellos mostraron $r: 0,77$; y ellas r: 0,75, $(p<0,01)$.

Tabla 2. Correlación entre el IMC y el \% de grasa de los sujetos evaluados.

\begin{tabular}{|c|c|c|}
\hline IMC & MASCULINO & FEMENINO \\
\hline Bajo Peso (M:52 - F:33) & 0,10 & $0,36\left(^{*}\right)$ \\
\hline Peso Ideal (M:188 - F: 175) & $0,48(* *)$ & $0,59\left({ }^{* *}\right)$ \\
\hline Sobrepeso (M: 18 - F: 31) & 0,37 & 0,31 \\
\hline Obesidad (M: 5 - F: 3) & 0,62 & 0,90 \\
\hline Total (M:263 - F:242) & $0,77\left(^{* \star}\right)$ & $0,75\left(^{* \star}\right)$ \\
\hline
\end{tabular}

M: Masculino; F: Femenino. ** La correlación es significativa al nivel 0,01 (bilateral).

De igual manera, en la tabla 3 se observan las correlaciones entre el IMC y el porcentaje de grasa corporal en los diferentes grupos de edades abordados para ambos sexos. Aquí se puede destacar que en la mayoría de los grupos, en ambos sexos, presentaron correlaciones altas y significativas $(p<0,01)$, con un coeficiente de determinación mayor al $50 \%$ en todos los grupos para el sexo masculino, excepto para el G3 de dicho sexo. Asimismo, en el gráfico 1 se destaca la relación lineal entre las variables, apreciándose un coeficiente de determinación mayor al 50\% para ambos sexos.

Rev. Ib. CC. Act. Fis. Dep. 
Tabla 3. Correlación entre el IMC y el \% de grasa por grupo de edades.

\begin{tabular}{lcc}
\hline GRUPO EDAD & MASCULINO & FEMENINO \\
\hline G1 (M:80 - F:91) & $0,81\left(^{* \star}\right)$ & $0,72\left(^{* \star}\right)$ \\
G 2 (M:93 - F:73) & $0,82\left(^{* *}\right)$ & $0,83\left(^{* \star}\right)$ \\
G3 (M:90 - F:78) & $0,66\left(^{* \star}\right)$ & $0,81\left(^{(\star}\right)$ \\
\hline
\end{tabular}

** La correlación es significativa al nivel 0,01 (bilateral). * La correlación es significativa al nivel 0,05 (bilateral).

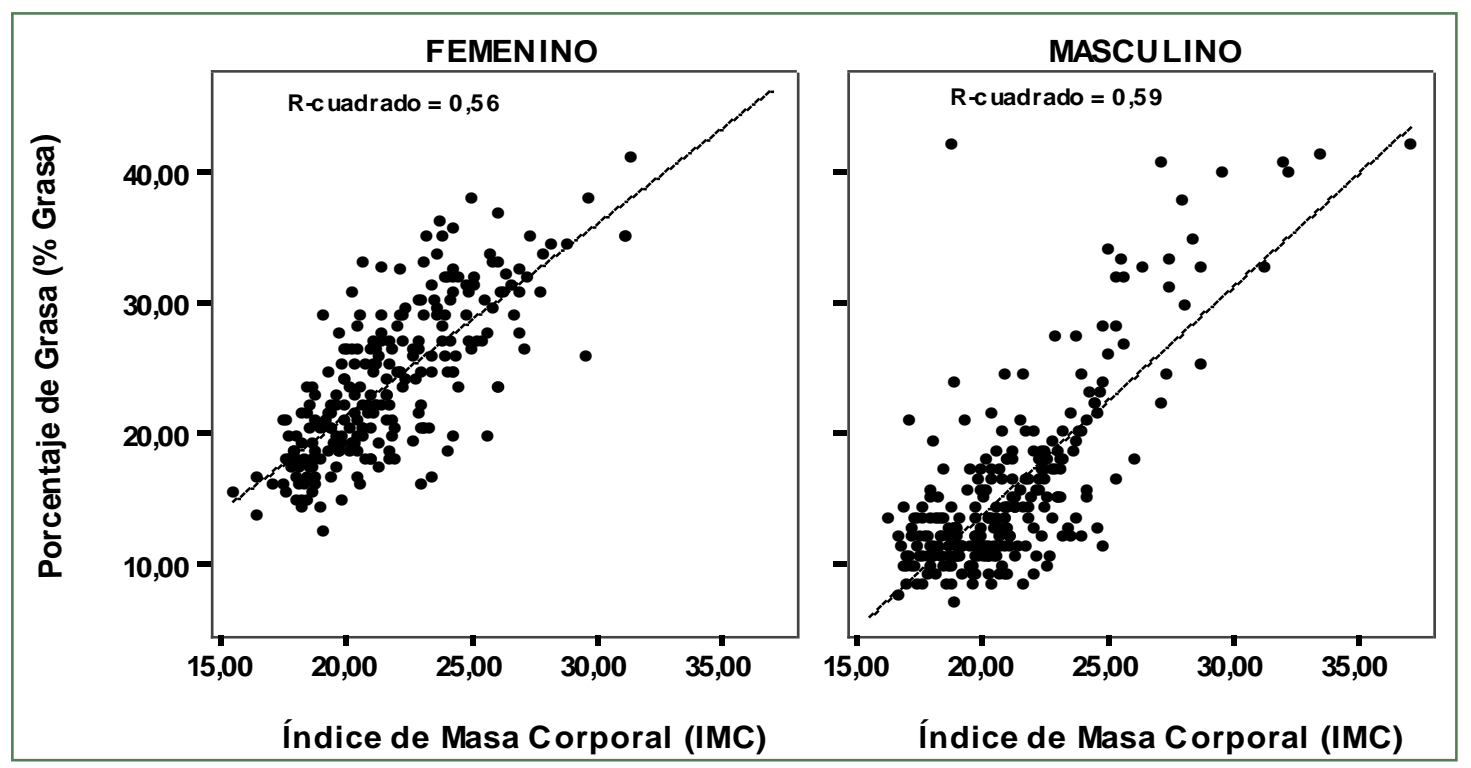

Gráfico 1. Diagrama de dispersión con la recta ajustada del modelo de regresión lineal simple de las variables porcentaje de grasa y el índice de masa corporal en estudiantes del liceo Bolivariano Guanipa.

\section{DISCUSIÓN}

La investigación tuvo como principal objetivo determinar la relación entre el índice de masa corporal y el porcentaje de grasa corporal en 505 estudiantes (242 del sexo femenino y 263 masculino) de un Liceo Bolivariano del estado Barinas, con edades comprendidas entre $10 \mathrm{y}$ 20 años. Los principales resultados revelan relaciones altas y significativas entre el IMC y el porcentaje de grasa corporal para ambos sexo y para los diversos grupos de edades en que fueron distribuidos los sujetos. Sin embargo, existen discrepancias al momento de su caracterización del IMC, en donde las correlaciones resultaron de bajas a moderadas.

A pesar de ello se considera al IMC como un indicador adecuado para estimar el nivel nutricional, ya que es fácil de calcular, y los valores de corte para niños se ajustan al sexo y la edad.

Rev. Ib. Cᄃ. Act. Fis. Dep.
Los resultados del presente estudio contrastan con los de ${ }^{(14)}$, quienes mostraron que el IMC explica el $73 \%$ y el $63 \%$ de la varianza de la grasa corporal en niños y niñas menores de 13 años, respectivamente, mientras que tiene menor valor predictivo en niños mayores. De acuerdo con los resultados de la presente investigación, el IMC fue más selectivo en la predicción del porcentaje de grasa en los niños, mientras que en las niñas fue algo inferior, contrariamente a los resultados de ${ }^{(15,16)}$, en donde la correlación entre el porcentaje de grasa y el IMC era más fuerte en las niñas. Asimismo, coinciden con los hallazgos presentados por ${ }^{(17)}$, quienes determinaron que la precisión al momento de clasificar utilizando los percentiles de IMC para identificar los grupos de riesgo en porcentaje de grasa oscilaron entre $86,9 \%$ y $89,1 \%$ para ambos sexos.

En resumen, el IMC se considera como una herramienta práctica para detectar el exceso de sobrepeso y adiposidad en niños, por lo que su uso común en clínicas, escuelas e 
investigaciones permitirá establecer claramente la concordancia con dichos parámetros antropométricos.

Por otra parte, la principal limitación que presentó el estudio radica fundamentalmente en su corte transversal, aunado a las diferencias obtenidas en la relación por las clasificaciones del IMC. Por último, hay que tener en cuenta que la relación entre el IMC y el porcentaje de grasa corporal se debe a múltiples factores, como lo son la madurez sexual, la raza, sexo y el patrón de distribución del tejido adiposo, lo cual debe considerarse para estudios longitudinales posteriores.

\section{CONCLUSIONES}

En función de los resultados obtenidos en la investigación se concluye que:

- Existen diferencia significativa entre ambos sexos para las variables estatura y el porcentaje de grasa corporal.

- Existe relación alta entre el IMC y el porcentaje de grasa corporal para ambos sexos y en los 3 subgrupos de edades en los cuales se subdividió la muestra en estudio, pertenecientes al Liceo Bolivariano Guanipa.

- EI IMC explica al porcentaje de grasa en los estudiantes evaluados, en todos los grupos de edades para ambos sexos, con un coeficiente de determinación mayor al $50 \%$.

\section{Aplicaciones prácticas}

Se recomienda la utilización del IMC para la estimación del estado nutricional en estudiantes del estado Barinas con características similares a la muestra utilizada en la presente investigación. Asimismo, utilizar los criterios de referencia del Obesity Task Force (IOTF) para la clasificación del IMC en poblaciones escolares. Llevar a cabo estudios longitudinales que permitan una evaluación continua del estado nutricional a través del IMC.

\section{Agradecimientos}

Especial agradecimiento a los profesores Ricardo Godoy, Norman Prieto y Rafael Martínez, por su ayuda en la recolección de los datos. Asimismo, a la dirección de la institución por el apoyo brindado en la logística. También agradecemos a los estudiantes que participaron en este estudio por su colaboración comprometida.

Financiación: Propia del autor. Conflicto de interés: Ninguno

\section{REFERENCIAS BIBLIOGRÁFICAS}

1. Kaufer, M. y Toussaint, G. Indicadores antropométricos para evaluar sobrepeso y obesidad en pediatría. Boletín médico del Hospital Infantil de México 2008; 65(6): 502-518.

2. OMS. 10 datos sobre la obesidad. Extraído el 02 de agosto de 2013 desde http://www.who.int/features/factfiles/obesity/es/\#.

3. FAO. América Latina, entre la obesidad y el hambre. Extraído el 02 de agosto de 2013 desde http://www.fao.org/agronoticias.

4. INN (2010). Obesidad infantil en Venezuela registra $9 \%$ de incidencia. Extraído el 02 de agosto de 2013 desde http://www.avn.info.ve/contenido/obesidadinfantil-venezuela-registra-9-incidencia.

5. González, M., Benito, P. y Meléndez, A. Obesidad 2008; En J. López Chicharro y I. López Mojares (Ed.), Fisiología clínica del ejercicio (pp. 279-300). España: Panamericana. Editorial.

6. Biljana, S., Borislav, O., Goran, D., Stokic, E. y Sinisa B. Relationship between body mass index and body fat in children-Age and gender differences. Obesity Research \& Clinical Practice 2012; 6(2): e167-e173.

7. Alvero-Cruz, JR., Carnero, E., Fernández-García, J.C., Barrera, J., Carrillo de Albornoz, M., \& Sardinha, L. (2010) Validez delos índices de masa corporal y de masa grasa como indicadores de sobrepeso en adolescentes españoles: estudio Esccola. Medicina Clínica, 135(1), 8-14

8. Freedman, D. y Bettylou, S. The Validity of BMI as an Indicator of Body Fatness and Risk Among Children. Oficial Journal of the American Academic of Pediatrics 2009; 124(1): 5234-534.

9. Rolland, M., Deheeger, M. y Bellisle, F. The adiposity rebound and adult obesity. In: Madeiros-Neto G, Halpern A, Bouchard C, editors 2008; Progress in obesity research: 9. Proceedings of the 9th International Congress on Obesity. [Documento en línea], Disponible en http://web.ebscohost.com. [Consulta: 2013, junio 10].

10. Demerath, E., Schubert, C., Maynard, M., Sun, S., Chumlea, W. y Pickoff, A. Do changes in body mass index percentile reflect changes in body composition in children? Data from the Fels Longitudinal Study. Oficial Journal of the American Academic of Pediatrics 2006; 117(3): e487-e495.

11. Slaughter, M., Lohman, T. Boileau, R., Horswill, C. Stillman, R. y Van Loan, M. Skinfold equations for estimation of body fatness in children and youth. Human Biology 1988; 60(5): 709-723.

12. Ordaz, E. Tratamiento de la información en cineantropometría. En D. Cabañas y F. Esparza (Ed.), Compendio de cineantropometría 2009; (pp. 105-118). España: CTO. Editorial.

13. Ruiz, B. Instrumentos de investigación educativa. Procedimientos para su diseño y validación. Venezuela: Centro de investigación y desarrollo en educación y gerencia 2002.

14. Widhalm, K., Schönegger, K., Huemer, C. y Auterith, A. Does the BMI reflect body fat in obese children and adolescents? A study using the TOBEC. International journal of obesity and related metabolic disorders 2001; 25(2): 279-285.

Rev. Ib. Cᄃ. Act. Fis. Dep. 
Relación del índice de masa corporal y el porcentaje de grasa corporal en jóvenes venezolanos

Padilla, ل.

15. Morimoto, A., Nishimura, R., Sano, H., Matsudaira, T., Miyashita, Y. y Shirasawa. T. Gender differences in the relationship between percent body fat (\%BF) and body mass index (BMI) in Japanese children. Diabetes Research and Clinical Practice 2007; 78(1): 123-125.

16. Pietrobelli, A., Faith, M., Allison, D., Gallagher, D., Chiumello, G. y Heymsfield, S. Body mass index as a measure of adiposity among children and adolescents: a validation study. Journal Pediatric 2006; 132(2): 204-210.

17. Laurson, K., Eisenmann, J. y Welk, G. Body Mass Index Standards Based on Agreement with Health-Related Body Fat. American Journal of Preventive Medicine 2011; 41(4S2): S100-S105.

Referencias totales citadas: 14 .

Referencias citadas correspondientes a la Rev Ib CC Act Fis Dep: 0.

Rev. Ib. CC. Act. Fis. Dep. 